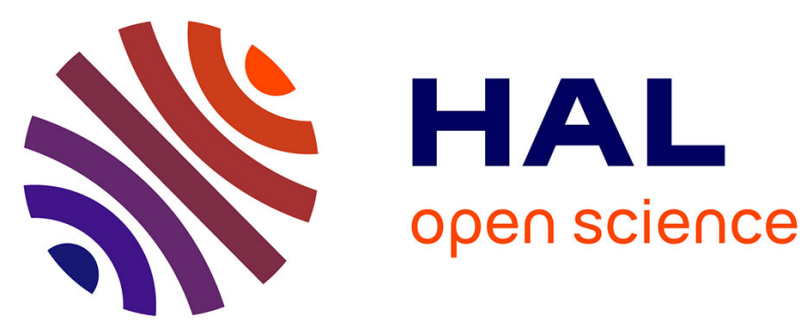

\title{
Hysteresis Photomodulation via Single-Crystal-to-Single-Crystal Isomerization of a Photochromic Chain of Dysprosium Single-Molecule Magnets
}

Maher Hojorat, Hassan Al Sabea, Lucie Norel, Kevin Bernot, Thierry Roisnel, Frédéric Gendron, Boris Le Guennic, Elzbieta Trzop, Eric Collet, Jeffrey

Long, et al.

\section{- To cite this version:}

Maher Hojorat, Hassan Al Sabea, Lucie Norel, Kevin Bernot, Thierry Roisnel, et al.. Hysteresis Photomodulation via Single-Crystal-to-Single-Crystal Isomerization of a Photochromic Chain of Dysprosium Single-Molecule Magnets. Journal of the American Chemical Society, 2020, 142 (2), pp.931-936. 10.1021/jacs.9b10584 . hal-02432642

\section{HAL Id: hal-02432642 \\ https://hal.science/hal-02432642}

Submitted on 13 Feb 2020

HAL is a multi-disciplinary open access archive for the deposit and dissemination of scientific research documents, whether they are published or not. The documents may come from teaching and research institutions in France or abroad, or from public or private research centers.
L'archive ouverte pluridisciplinaire HAL, est destinée au dépôt et à la diffusion de documents scientifiques de niveau recherche, publiés ou non, émanant des établissements d'enseignement et de recherche français ou étrangers, des laboratoires publics ou privés. 


\title{
Hysteresis Photomodulation via Single-Crystal-to-Single-Crystal Isomerization of a Photochromic Chain of Dysprosium Single-Mole- cule Magnets
}

\author{
Maher Hojorat,${ }^{\dagger}$ Hassan Al Sabea, ${ }^{\dagger}$ Lucie Norel, ${ }^{\dagger *}$ Kevin Bernot, ${ }^{\dagger *}$ Thierry Roisnel, ${ }^{\dagger}$ Frederic Gen- \\ dron, ${ }^{\dagger}$ Boris Le Guennic, ${ }^{\dagger}$ Elzbieta Trzop,${ }^{\ddagger}$ Eric Collet, ${ }^{\dagger}$ Jeffrey R. Long $,{ }^{\S}, \|, \#$ and Stéphane Rigaut ${ }^{\dagger}$ \\ †Univ Rennes, INSA Rennes, CNRS, ISCR (Institut des Sciences Chimiques de Rennes) - UMR 6226, F-35000 Rennes, \\ France \\ Univ Rennes, CNRS, IPR (Institut de Physique de Rennes) - UMR 6251, F-35000 Rennes, France \\ $\S$ Department of Chemistry and "Department of Chemical and Biomolecular Engineering, University of California, Berkeley, \\ California 94720, United States \\ \#Materials Sciences Division, Lawrence Berkeley National Laboratory, Berkeley, California 94720, United States
}

KEYWORDS : Photochromism, Single Molecule Magnet, Dysprosium, Photocrystallography, Ab Initio Calculations

\begin{abstract}
A one-dimensional coordination solid is synthesized by reaction of a bispyridyl dithienylethene (DTE) photochromic unit with the highly anisotropic dysprosium-based single-molecule magnet [Dy(Tppy)F(pyridine)2]PF6. Slow magnetic relaxation characteristics are retained in the chain compound, $1 \mathrm{c}$, and photoisomerization of the bridging DTE ligand induces a single-crystal-to-single-crystal transformation that can be monitored using photocrystallography. Notably, the resulting chain compound 1o exhibits faster low-temperature relaxation than $1 \mathrm{c}$, which is apparent in magnetic hysteresis data collected for both compounds as high as $4 \mathrm{~K}$. Ab initio calculations suggest that this photomodulation of the magnetic relaxation behavior is due to crystal packing changes rather than changes to the crystal field splitting upon ligand isomerization.
\end{abstract}

\section{Introduction}

Molecules that exhibit slow magnetic relaxation resulting from a bistable magnetic ground state are collectively known as singlemolecule magnets, and such systems stand as attractive candidates for next generation information storage schemes. ${ }^{1-2}$ Mononuclear single-molecule magnets of the lanthanides are some of the most promising compounds investigated to date, ${ }^{3-6}$ and they can exhibit $U_{\text {eff values }}{ }^{7-9}$ exceeding $1000 \mathrm{~cm}^{-1}$ originating from splitting of the ground state by the crystal field, as well as record blocking temperatures. ${ }^{7-9}$ Efficient control of their magnetization via an external stimulus - such as electric potential, ${ }^{10-12}$ pressure, coordinating solvent, ${ }^{13}$ temperature, ${ }^{14}$ or light ${ }^{15}$ - could considerably increase the scope of applications for these magnetic molecules. Light is particularly intriguing as a tool for this purpose, as it could potentially enable remote and rapid control of magnetic characteristics. The first example of a system exhibiting light-induced on-off switching of single-molecule magnet behavior was the spin-crossover complex $\left[\mathrm{Fe}(\mathrm{ptz})_{6}\right]\left(\mathrm{BF}_{4}\right)_{2}(\mathrm{ptz}=1$-propyltetrazole), in which iron(II) converts from a low spin to a high spin configuration under visible light irradiation. ${ }^{16-17}$ We are interested in creating similar photoswitching behavior in intrinsically non-photo-responsive mononuclear lanthanide single-molecule magnets, and we have therefore been targeting systems featuring photoactive ligands.

Indeed, given the importance of the coordination environment in dictating magnetic anisotropy and relaxation behavior in $4 \mathrm{f}$ systems, successful photo control of ligand structure may provide an efficient handle for external manipulation of these properties. Previous efforts to design magnetic molecules or chains featuring photoswitchable ligands, ${ }^{18-22}$ including by some of us, ${ }^{23-24}$ revealed a number of challenges inherent to this approach, including lack of photochromism, ${ }^{20,}{ }^{23}$ poor reversibility, ${ }^{22}$ changes in crystallinity upon irradiation that preclude precise magnetostructural correlations, ${ }^{25}$ and finally, the occurrence of slow relaxation only at very low temperatures and the absence of magnetic hysteresis. ${ }^{18,24}$
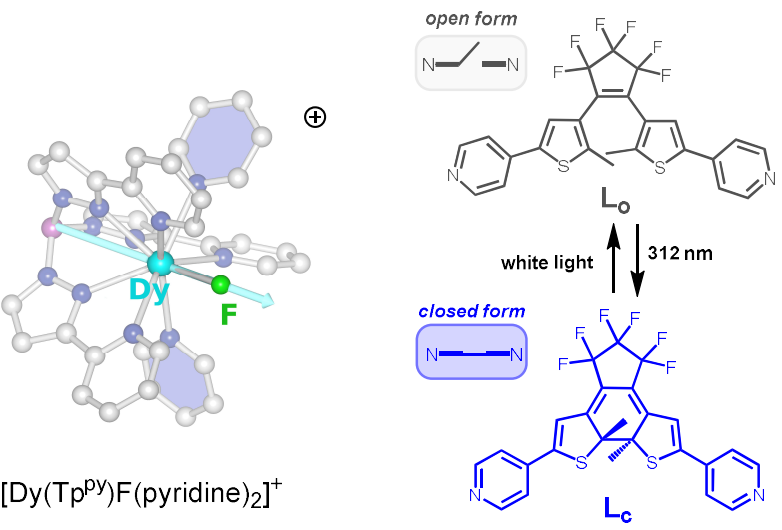

Figure 1. (Left) Molecular structure of the cation in $\left[\mathrm{Dy}\left(\mathrm{Tp}^{\mathrm{py}}\right) \mathrm{F}\right.$ (pyridine)2]PF used in assembling the one-dimensional chain compound, $\mathbf{1}$, in this work. Grey, blue, purple, pale blue, and green spheres represent C, N, B, Dy, and F atoms, respectively. (Right) Open (top) and closed (bottom) forms of the photoactive bispyridyl dithienylethene unit used as a bridging ligand in $\mathbf{1}$.

Here, we present a supramolecular strategy to obtain a photoswitchable chain of lanthanide single-molecule magnets featuring $\left[\mathrm{Dy}\left(\mathrm{T} \mathrm{p}^{\mathrm{py}}\right) \mathrm{F} \text { (pyridine) }\right)_{2} \mathrm{PF}_{6}{ }^{26}\left(\mathrm{Tp}^{\mathrm{py}}=\operatorname{tris}(3-(2-\right.$ pyridyl $)$ pyrazolyl)hydroborate) as a building unit. This complex was previously 
shown to exhibit slow magnetic relaxation at high temperatures as a result of strong axial magnetic anisotropy generated by a short Dy- $\left(\eta^{1}-\mathrm{F}\right)$ bond. ${ }^{27}$ Exchange of the equatorial pyridine ligands in this compound with a ditopic photoactive bispyridyl dithienylethene (DTE) ligand ${ }^{28}$ yields infinite chains of single-molecule magnets that undergo a single-crystal-to-single-crystal transformation upon visible irradiation and exhibit unprecedented photomodulation of magnetic hysteresis behavior at temperatures as high as $4 \mathrm{~K}$.

\section{Results and Discussion}

Synthesis and Structural Characterization. In our design of a photoswitchable lanthanide-based molecular magnet, we chose bispyridyl dithienylethene as a linker given that the DTE unit exhibits excellent fatigue resistance in the presence of prolonged light exposure, facile photoisomerization in the solid state, and high isomerization quantum yields. ${ }^{29}$ In particular, the open form of bispyridyl dithienylethene ( $\left.\mathbf{L}_{\mathbf{o}}\right)$ undergoes UV-light driven photoisomerization to the stable closed isomer $\left(\mathbf{L}_{\mathbf{c}}\right)$, and this process is reversible under visible light (Figure 1) ${ }^{28}$ We obtained the chain compound $\left[\mathrm{Dy}\left(\mathrm{Tp} \mathrm{p}^{\mathrm{py}}\right) \mathrm{F}\left(\mathbf{L}_{\mathbf{c}}\right)\right] \mathrm{PF}_{6}\left(\mathbf{1}_{\mathbf{c}}\right)$ by layering a solution of $\left[\mathrm{Dy}\left(\mathrm{Tp}^{\mathrm{py}}\right) \mathrm{F}\right.$ (pyridine) 2$] \mathrm{PF}_{6}$ dissolved in dichloromethane with a dichloromethane solution of $\mathbf{L} \mathbf{c}$. Storage of this solution mixture at room temperature overnight resulted in the formation of dark blue prism-shaped crystals of $\mathbf{1}_{\mathbf{c}}$ (Figure 2). In contrast, attempts to repeat the same crystallization procedure with $\mathbf{L}_{\mathbf{o}}$ did not result in the formation of any solid material, even after several days.

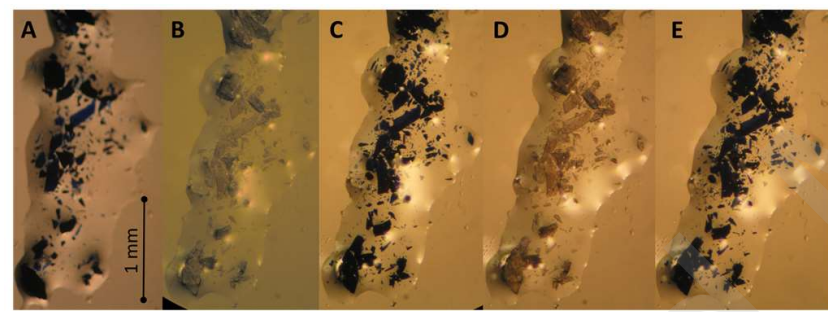

Figure 2. Photographs of crystals of $\mathbf{1}_{\mathbf{c}}$ in paratone oil: initial state (A), after $6 \mathrm{~h}$ white light irradiation (B) and subsequent $25 \mathrm{~min}$ UV light irradiation $(\mathrm{C})$ and second cycle of $6 \mathrm{~h}$ white light irradiation (D) and 25 min UV light irradiation.

Compound $\mathbf{1}$ strongly absorbs in the visible range and its absorption spectrum, recorded using a $\mathrm{KBr}$ pellet, showed transitions characteristic of the DTE ligand in its closed state, with a broad band centered at $\lambda_{\max }=600 \mathrm{~nm}$ (see Figure S2), consistent with that of the free ligand in solution $\left(\lambda_{\max }=592 \mathrm{~nm}\right) .{ }^{28}$ After white light irradiation, the pellet was totally bleached and only UV transitions below $350 \mathrm{~nm}$ were observed. The blue color could be restored upon irradiation with $365 \mathrm{~nm}$ light, and the process was reversible over several cycles of bleaching and coloring, with no apparent photodegradation (see Figure S3). Similarly, crystals of $\mathbf{1}_{\mathbf{c}}$ could be bleached upon white light irradiation and their blue color was restored upon $365 \mathrm{~nm}$ irradiation (Figure 2).

Single-crystal x-ray diffraction characterization of blue $\mathbf{1}_{\mathbf{c}}$ at $300 \mathrm{~K}$ revealed that this compound crystallizes in the monoclinic space group $P 2{ }_{1} / c$ (Table $\mathrm{S} 1$ ) and is composed of $\left[\mathrm{DyF}\left(\mathrm{Tp}^{\mathrm{py}}\right)\right]^{+}$units bridged by bidentate $\mathbf{L}_{\mathbf{c}}$ ligands (Figure 3 ). It also contains large pores, probably filled by dichloromethane molecules that could not be identified and modeled further because of the important disorder within the pores. Importantly, the very short Dy-F bond (2.098(4) $\AA$ ) is preserved upon exchange of the pyridine ligand with bispyridyl dithienylethene, and each dysprosium ion is nine-coordinate with a coordination sphere best described as a spherical capped square antiprism, ${ }^{30}$ very similar to that of the parent mononuclear compound (Tables S2 and S3). The DTE linker in $\mathbf{1}_{\mathbf{c}}$ shows geometrical features typical of the closed isomer, and the existence of two enantiomers with opposite configurations of the chiral carbons leads to crystallographic disorder in the central ring.

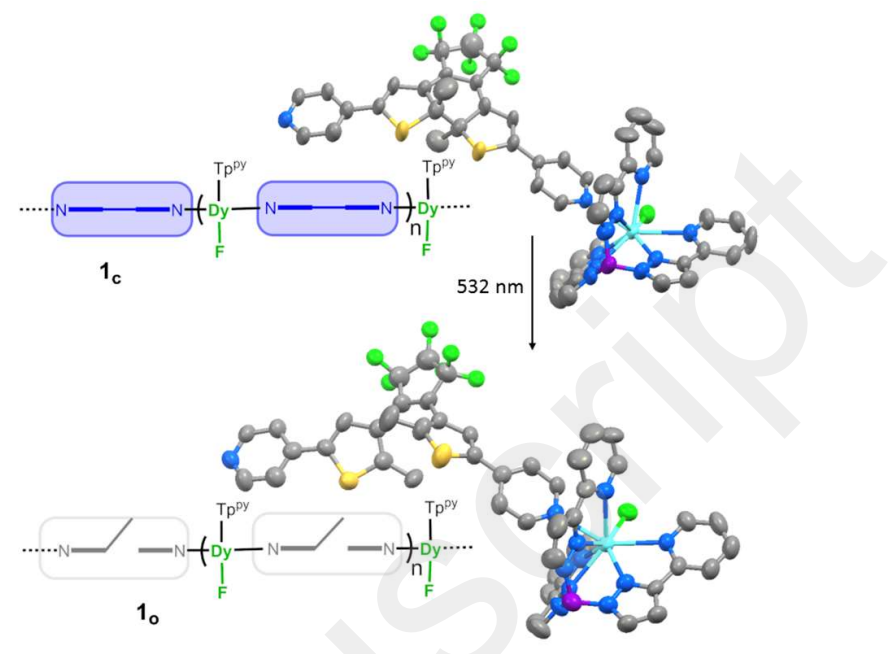

Figure 3. Single-crystal $x$-ray diffraction structures of the asymmetric units of compound 1 $1 \mathrm{c}$ at $300 \mathrm{~K}$ (top) and $\mathbf{1}_{\mathrm{o}}$ at $293 \mathrm{~K}$ (bottom). Grey, blue, purple, green, yellow and light blue ellipsoids represent C, N, B, F, S and Dy atoms, respectively; $\mathrm{H}$ atoms and $\mathrm{PF}_{6}{ }^{-}$counter ions are not shown.

Photocrystallography. Irradiation of a single crystal of $\mathbf{1}_{\mathbf{c}}$ with green light ( $\left.532 \mathrm{~nm}, 4 \mathrm{~mW} \cdot \mathrm{mm}^{-2}\right)$ resulted in a color change from dark blue to colorless (Figure 4, inset) and variation of the unit cell parameters resulting in an overall volume increase (Table S1). A photostationary state corresponding to a fully bleached crystal was reached after four hours of irradiation. The irradiated product, $\mathbf{1}_{\mathbf{0}}$, also crystallizes in $P 2{ }_{1} / c$ (see Table S1) with a molecular structure that is very similar to $\mathbf{1}_{\mathbf{c}}$, while in $\mathbf{1}_{\mathrm{o}}$ the bridging DTE ligand adopts its open form. This ring opening results in a slight chain elongation and consequently a small increase in the $b$ and $c$ lattice parameters of $\mathbf{1}_{\mathrm{o}}$ relative to $\mathbf{1}_{\mathrm{c}}(1.3 \%$ and $2.75 \%$, respectively). In contrast, the lattice parameter $a$ decreases by $1.5 \%$ in $\mathbf{1}_{\mathrm{o}}$ relative to $\mathbf{1}$, as a result of closer interchain contacts (Figure S7-S8). Importantly, in $\mathbf{1}_{\mathbf{0}}$ the distance between the opposing carbon atoms of the thiophene rings is $3.437(19) \AA$ (c.f. 1.450(11) and 1.43(5) $\AA$ for the two positions in $\mathbf{1}_{\mathrm{c}}$ ), which is below the maximum threshold of $4.2 \AA$ above which photocyclization is not possible in the solid state. ${ }^{29,31}$ Gratifyingly, irradiation with $365 \mathrm{~nm}$ light results in darkening of the single crystal, suggesting a reversible photocyclization reaction of $\mathbf{1}_{\mathbf{0}}$ toward 1c (Figure 3) in solid state. However, the slight change in the lattice parameters indicates only partial conversion (Figure 4), which may be due to limited bulk photoexcitation owing to crystal darkening. Over the course of irradiation, we noted gradual loss of crystallinity and $\mathrm{x}$-ray diffraction signal, which precluded further structural analysis. 


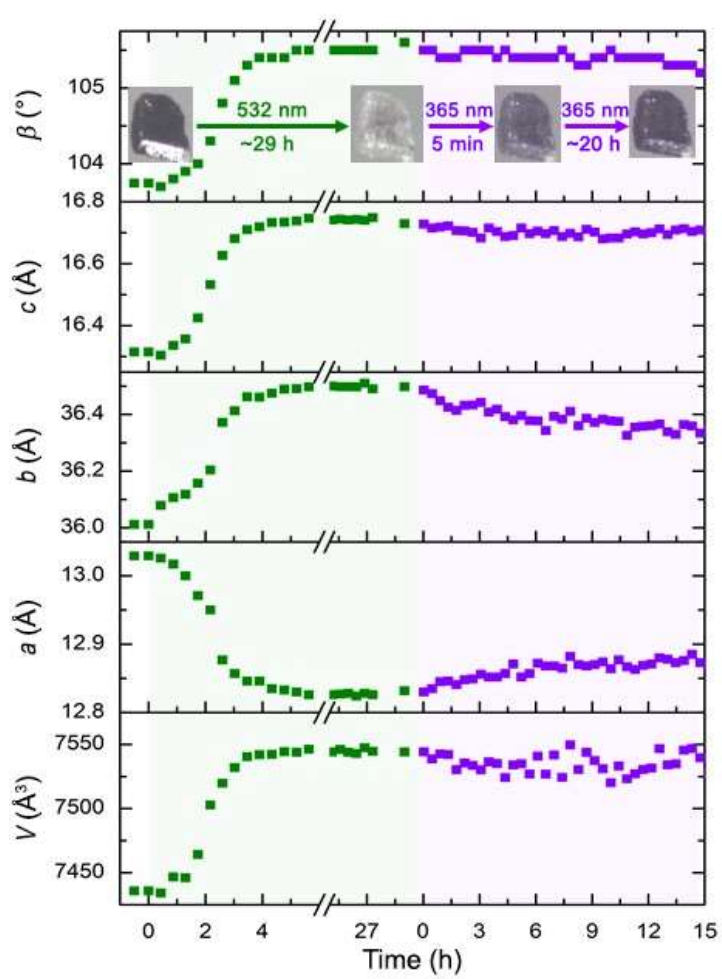

Figure 4. Evolution of the unit cell parameters of a starting crystal of $\mathbf{1}_{\mathbf{c}}$ upon continuous irradiation with $532 \mathrm{~nm}$ light (green dots) followed by $365 \mathrm{~nm}$ light (purple dots). (Inset) images of the single crystal used in the photocrystallographic study.

Closer examination of the unit cell changes upon photoisomerization of $\mathbf{1}_{\mathrm{c}}$ to $\mathbf{1}_{\mathbf{o}}$ reveals a non-linear response to light excitation. Indeed, very little change occurs in the first hour of irradiation, but after a latency period of $\sim 2 \mathrm{~h}$ the crystal volume expands more rapidly, and full conversion to $\mathbf{1}_{\mathrm{o}}$ is achieved within $\sim 3 \mathrm{~h}$. This response indicates the existence of positive feedback associated with ring opening and molecular packing, as initial bond cleavage and volume expansion promotes further ring opening. This response to light excitation is in contrast to that of more conventional spin crossover materials, in which each absorbed photon switches an isolated molecule and the lattice packing has a less pronounced effect on photoinduced transformation. ${ }^{32-33}$ As noted above, isomerization of $\mathbf{1}_{\mathbf{o}}$ in the single crystal is shown to be much less efficient than for $\mathbf{1}$, which could be a result of a more favorable packing rearrangement in the open form. For example, stabilizing intermolecular contacts between the fluorine and sulfur atoms of the DTE unit and one pyridylpyrazolyl arm might render photocyclization more difficult to achieve (Figure S9). Although DTE isomerization in single crystals is known, ${ }^{34}$ to our knowledge compound $\mathbf{1} \mathbf{c}$ is one of only a few examples ${ }^{25,35}$ of compounds exhibiting a full transformation between open and closed states, which enables accurate crystal structure determination and magneto-structural correlations.

Ab initio Calculations. We sought to further investigate the structural differences between compounds $\mathbf{1}_{\mathbf{c}}$ and $\mathbf{1}_{\mathbf{o}}$, given that changes to the dysprosium coordination sphere can affect the molecular ground state crystal field splitting (and consequently the magnetic relaxation barrier and other mechanisms of slow relaxation), while changes in Dy $\cdots$ Dy distances can impact dipolar interactions. Analysis of $\mathbf{1}_{\mathrm{c}}$ and $\mathbf{1}_{\mathrm{o}}$ using the continuous shape measures approach $^{30}$ (see Table S3) revealed that the dysprosium coordination spheres can be described as approximate capped square antiprisms, with minor variations between them. Ab initio calculations supported this analysis (Figure S21) and revealed that, relative to the parent molecular complex, the short Dy-F bond remains largely unaffected in both chain compounds, as does the energy splitting of the $J=15 / 2$ ground state and the nearly perfect axial $M_{J}$ $= \pm 15 / 2$ ground Kramers doublet (Table S13). Closer inspection of the extended structures of $\mathbf{1}_{\mathrm{c}}$ and $\mathbf{1}_{\mathrm{o}}$ revealed three characteristic Dy $\cdots$ Dy distances, identified in Figure $\mathrm{S} 10$ as $\mathrm{d}_{1}, \mathrm{~d}_{2}$, and $\mathrm{d}_{3}$, corresponding to nearest and next-nearest interchain dysprosium neighbors and nearest-neighbor intrachain dysprosium ions, respectively. Upon conversion from $\mathbf{1}_{\mathbf{c}}$ to $\mathbf{1}_{\mathbf{o}}$, the most significant changes occur for $\mathrm{d}_{2}(\sim 11.2 \rightarrow 11.7 \AA)$ and $\mathrm{d}_{3}(\sim 18.9 \rightarrow 19.4 \AA)$. Calculated dipolar coupling constants $\left(J^{\text {Dip }}\right)$ indicate that antiferromagnetic interactions occur between dysprosium ions related by $\mathrm{d}_{1}$, and ferromagnetic interactions occur between dysprosium ions related by $\mathrm{d}_{2}$ and $\mathrm{d}_{3}$, although the individual constants are all small in magnitude and differ only slightly between compounds (Table S14).

Influence of photoisomerization on the magnetic properties. Static (dc) and dynamic (ac) magnetization data were collected for a microcrystalline pellet of $\mathbf{1}_{\mathbf{c}}$ to evaluate whether slow magnetic relaxation persists in this system upon ligand exchange and coordination polymer formation when compared with $\left[\mathrm{Dy}\left(\mathrm{Tp}^{\mathrm{py}}\right) \mathrm{F}(\right.$ pyridine) $)_{2} \mathrm{PF}_{6}$. The molar magnetic susceptibility-temperature product $\left(\chi_{\mathrm{M}} T\right)$ for $\mathbf{1}_{\mathrm{c}}$ at $298 \mathrm{~K}$ is $13.94 \mathrm{emu} \mathrm{K} \mathrm{mol}{ }^{-1}$ (Figure S11), slightly larger than the value of $13.64 \mathrm{emu} \mathrm{K} \mathrm{mol}^{-1}$ for $\left[\mathrm{Dy}\left(\mathrm{Tp} \mathrm{p}^{\mathrm{py}}\right) \mathrm{F}(\mathrm{pyri}-\right.$ dine) $)_{2} \mathrm{PF}_{6}$ and in good agreement with the predicted value of 14.17 emu K mol${ }^{-1}$ for an isolated $\mathrm{Dy}^{3+}$ ion $\left({ }^{6} \mathrm{H}_{15 / 2}\right)$. Ac magnetic susceptibility data revealed that $\mathbf{1}_{\mathbf{c}}$ indeed exhibits slow magnetic relaxation under zero applied dc field $\left(H_{\mathrm{ac}}=3 \mathrm{Oe}, 1-1500 \mathrm{~Hz}\right.$, Figures S15-S18), with clear peaks in the out-of-phase susceptibility $\left(\chi^{\prime \prime}\right)$ as high as $44 \mathrm{~K}$ (c.f. $40 \mathrm{~K}$ for $\left[\mathrm{Dy}\left(\mathrm{Tp}^{\mathrm{py}}\right) \mathrm{F}\right.$ (pyridine) $\left.{ }_{2}\right] \mathrm{PF}_{6}$ ). Fits to the ac susceptibility data using a generalized Debye equation (Figure S20) relaxation times (Tables S4 and S8) that were used to generate the Arrhenius plots shown in Figure 5. Below $5 \mathrm{~K}$, the relaxation becomes temperature independent for both compounds, indicating quantum tunneling of the magnetization, while at higher temperatures there is more pronounced curvature in the data, suggesting a competition between Raman and Orbach magnetic relaxation processes on $\mathbf{1}_{\mathfrak{c}}$, as observed for $\left[\mathrm{Dy}\left(\mathrm{Tp}^{\mathrm{py}}\right) \mathrm{F}(\text { pyridine })_{2}\right] \mathrm{PF}_{6}$. These relaxation data can be fit using eq 1 :

$$
\tau^{-1}=B T^{n}+\tau 0^{-1} \exp \left(-U_{\mathrm{eff}} / k_{\mathrm{B}} T\right)+\tau_{\mathrm{unnel}}{ }^{-1}
$$

which includes terms for Raman relaxation ( $B$ is the Raman constant) and quantum tunneling of the magnetization, in addition to Orbach relaxation. To avoid overparameterization, we fit the relaxation times above $35 \mathrm{~K}$ assuming purely Orbach relaxation. For $\mathbf{1} \mathbf{c}$, we found $U_{\text {eff }}=157 \mathrm{~cm}^{-1}\left(\tau_{0}=7.7 \times 10^{-7} \mathrm{~s}\right)$, half the value previously determined for $\left[\mathrm{Dy}\left(\mathrm{Tp}^{\mathrm{py}}\right) \mathrm{F}(\text { pyridine })_{2}\right] \mathrm{PF}_{6}\left(336 \mathrm{~cm}^{-1}\right)$. The full temperature range $(2-44 \mathrm{~K})$ is fit with the Orbach parameters fixed in eq. 1 that affords $B=3.05 \mathrm{~s}^{-1} \cdot \mathrm{K}^{-n}, n=1.53$ and $\tau_{\text {tunnel }}=$ $0.0337 \mathrm{~s}$.

Similarly, comprehensive magnetic data were collected for a sample of $\mathbf{1}_{\mathrm{o}}$ to further investigate the effects of ligand isomerization on the dysprosium slow magnetic relaxation behavior. The sample of $\mathbf{1}_{\mathbf{0}}$ was obtained from continuous white light irradiation of crystals of $\mathbf{1}_{\mathbf{c}}$ over 30 hours, until they were completely colorless (Figure S4). Overall, the magnetic behavior of $\mathbf{1}_{\mathbf{0}}$ is very similar to that of $\mathbf{1}_{\mathbf{c}}$ and reminiscent of [ $\mathrm{Dy}\left(\mathrm{Tp} \mathrm{p}^{\mathrm{py}}\right) \mathrm{F}$ (pyridine) $\left.{ }_{2}\right] \mathrm{PF}_{6}$, thus only major features and relevant differences are detailed below (see the Supporting Information for complete magnetic analysis of $\mathbf{1}_{\mathbf{o}}$ and $\mathbf{1}_{\mathbf{c}}$ ). Analogous to $\mathbf{1}_{\mathbf{c}}$, chain $\mathbf{1}_{\mathbf{o}}$ exhibits slow magnetic relaxation under zero de field up to $44 \mathrm{~K}$ (Figure S15-S18). The same fitting procedure as the one for compound $\mathbf{1}_{\mathbf{c}}$ was used and we fit the relaxation times for $\mathbf{1}_{\mathrm{o}}$ above $35 \mathrm{~K}$ assuming purely Orbach relaxation, which yielded the same values of $U_{\text {eff }}\left(157 \mathrm{~cm}^{-1}\right)$ and $\tau_{0}(7.7$ $\times 10^{-7} \mathrm{~s}$ ). The most striking effect of the photoswitching between compounds is seen for the $\tau_{\text {tunnel }}$ value, which decreases from 
$0.0337 \mathrm{~s}$ for $\mathbf{1}_{\mathrm{c}}$ to $0.0015 \mathrm{~s}$ for $\mathbf{1}_{\mathbf{0}}$. Quantum tunneling is partially suppressed for both compounds under an optimal applied field of 1200 Oe (Figures 5 and S14), although data collected under this field indicate that magnetic relaxation remains faster for $\mathbf{1}_{0}$ than for $\mathbf{1}_{\mathbf{c}}$ in the temperature range where the Raman and tunneling mechanisms are dominant (2-20 K, see Table S10).

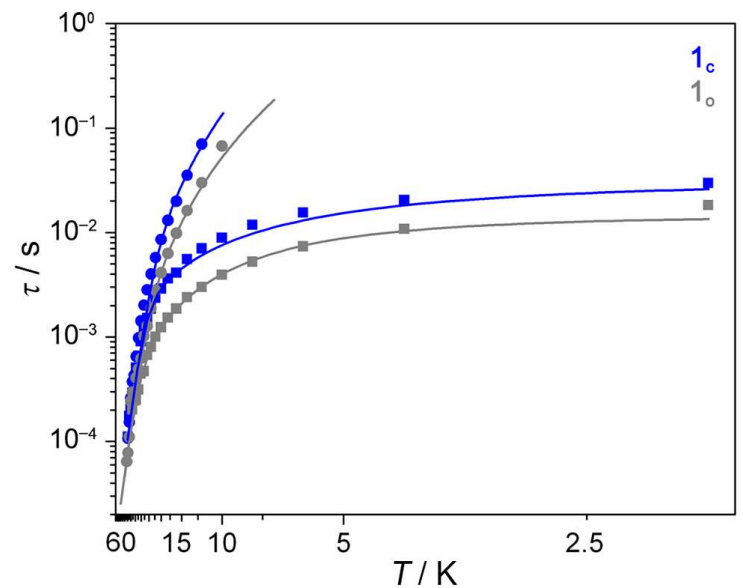

Figure 5. Plot of the relaxation time, $\tau$, (log scale) versus $T$ (inverse scale) for $\mathbf{1}_{\mathbf{c}}$ (blue) and $\mathbf{1}_{\mathbf{o}}$ (grey) measured under $H_{\mathrm{dc}}=0 \mathrm{Oe}$ (squares) and $H_{\mathrm{dc}}=1200 \mathrm{Oe}$ (dots). Lines are best fits according to eq 1 assuming fixed values of $U_{\text {eff }}=157 \mathrm{~cm}^{-1}$ and $\tau_{0}=7.7 \times 10^{-7} \mathrm{~s}$ for both compounds (see Table S12).

Variable-field magnetization data collected for $\mathbf{1}_{\mathbf{c}}$ and $\mathbf{1}_{\mathbf{o}}$ below $4 \mathrm{~K}$ revealed the presence of butterfly magnetic hysteresis, similar to that seen for the parent compound and many other dysprosiumbased single-molecule magnets. However, $\mathbf{1}_{\mathbf{c}}$ notably exhibits a wider hysteresis loop than $\mathbf{1}_{\mathbf{0}}$ at all investigated temperatures (Figures 6 and S12-S14). The slower relaxation for $\mathbf{1}_{\mathbf{c}}$ relative to $\mathbf{1}_{\mathbf{o}}$ could be due to differences in dipolar or spin-phonon coupling in each compound. For example, while the energy level splitting within $J=15 / 2$ is essentially the same for both chains, ab initio calculations indicate that subtle changes in the crystal packing induce a slightly stronger nearest-neighbor dipolar coupling in $\mathbf{1}_{\mathbf{o}}$ than in $\mathbf{1}_{\mathbf{c}}$ (vide supra). Unfortunately, attempts at site dilution in both chains using $\mathrm{Y}^{\mathrm{III}}, \mathrm{La}^{\mathrm{III}}$, or $\mathrm{Lu}^{\mathrm{III}}$ ions were unsuccessful, precluding efforts to examine the effects of dipolar interactions in these materials. We note that changes to the bridging ligand and molecular packing upon conversion from $\mathbf{1}_{\mathbf{c}}$ to $\mathbf{1}_{\mathbf{o}}$ may also influence the phonon bath and give rise to differences in spin-phonon coupling that manifest as differences in the magnetic hysteresis data. ${ }^{36-37}$ Finally, the structure being porous, reorganization of the solvent molecules within the pores could change slightly the electrostatic potential felt by the magnetic centers and affect the slow relaxation, an hypothesis that we cannot evaluate further since XRD data do not allow a precise identification and location of these solvent molecules

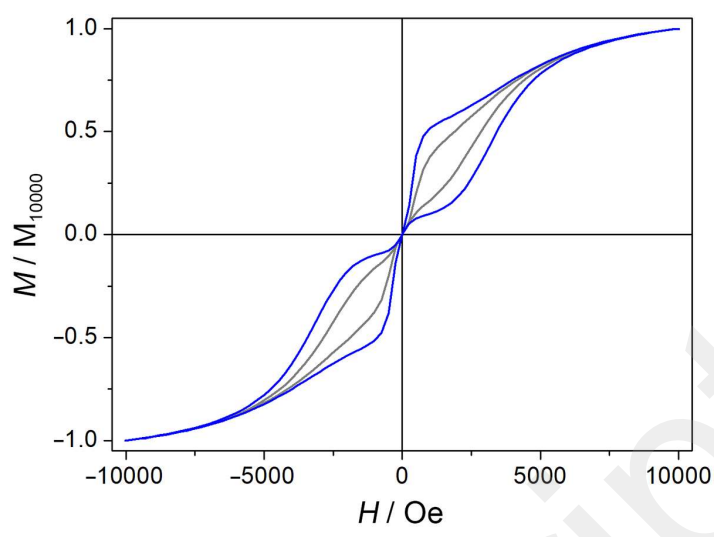

Figure 6. Variable-field magnetization data for $\mathbf{1}_{\mathbf{c}}$ (blue) and $\mathbf{1}_{\mathbf{o}}$ (grey) collected at $2 \mathrm{~K}$ using a field sweep rate of $15.5 \mathrm{Oe} \mathrm{min}^{-1}$. Data normalized on the basis of the magnetization value measured at 10000 Oe.

\section{Conclusion}

We have demonstrated an effective and generalizable synthetic approach to organize air-stable and highly anisotropic complexes, featuring short Dy- $\left(\eta^{1}-F\right)$ bonds, into a one-dimensional coordination solid, $\mathbf{1}_{\mathbf{c}}$, built up using photoactive DTE-based bridging ligands. Single-crystal-to-single-crystal photoizomerization of $\mathbf{1}_{\mathbf{c}}$ results in formation of $\mathbf{1}_{\mathbf{0}}$, featuring the open form of the DTE bridges. Both chain compounds exhibit slow magnetic relaxation with very similar behavior at high temperatures, reminiscent of the parent mononuclear complex. Below $5 \mathrm{~K}$, differences in magnetic hysteresis behavior are clear for $\mathbf{1}_{\mathbf{c}}$ and $\mathbf{1}_{\mathbf{o}}$, and can likely be ascribed to subtle differences in dipolar and spin-phonon coupling interactions. This work marks the first example of successful photomodulation of magnetic hysteresis behavior in a lanthanide-based system, and we are currently seeking to maximize this photomodulation effect through crystal engineering.

\section{ASSOCIATED CONTENT}

\section{Supporting Information}

The Supporting Information is available free of charge on the ACS Publications website. Experimental details for synthesis, single crystal XRD study, magnetic measurements, theoretical calculations (pdf) and cif files.

\section{AUTHOR INFORMATION}

\section{Corresponding Author}

E-mail: lucie.norel@univ-rennes1.fr, kevin.bernot@insarennes.fr.

Notes

The authors declare no competing financial interests.

\section{Funding sources}

This project received funding from the European Union's Horizon 2020 research and innovation program (Marie Curie grant agreement $\mathrm{N}^{\circ}$ 800719). We thank the Universite de Rennes 1 and the CNRS. K.B acknowledges the Institut Universitaire de France (IUF). B.L.G. and F.G. thank the French GENCI/IDRIS-CINES centers for high-performance computing resources. F.G. acknowledges Région Bretagne for a SAD Grant. J.R.L was supported under NSF grant CHE-1464841.

\section{ACKNOWLEDGMENT}


We thank Dr. K. R. Meihaus for editorial assistance.

\section{REFERENCES}

(1) Gatteschi, D.; Sessoli, R.; Villain, J., Molecular Nanomagnets. Oxford University Press: Oxford, 2006.

(2) Sanvito, S., Molecular spintronics. Chem. Soc. Rev. 2011, 40 (6), 3336-3355.

(3) Liu, J.-L.; Chen, Y.-C.; Tong, M.-L., Symmetry strategies for high performance lanthanide-based single-molecule magnets. Chem. Soc. Rev., 2018,47, 2431-2453.

(4) Rinehart, J. D.; Long, J. R., Exploiting single-ion anisotropy in the design of f-element single-molecule magnets. Chem. Sci. 2011, 2 (11), 2078-2085.

(5) Ishikawa, N.; Sugita, M.; Ishikawa, T.; Koshihara, S.; Kaizu, Y., Lanthanide double-decker complexes functioning as magnets at the singlemolecular level. J. Am. Chem. Soc. 2003, 125 (29), 8694-8695.

(6) Woodruff, D. N.; Winpenny, R. E. P.; Layfield, R. A., Lanthanide Single-Molecule Magnets. Chem. Rev. 2013, 113 (7), 5110-5148.

(7) Guo, F.-S.; Day, B. M.; Chen, Y.-C.; Tong, M.-L.; Mansikkamäki, A.; Layfield, R. A., A Dysprosium Metallocene Single-Molecule Magnet Functioning at the Axial Limit. Angew. Chem. Int. Ed. 2017, 56 (38), 11445-11449.

(8) Guo, F.-S.; Day, B. M.; Chen, Y.-C.; Tong, M.-L.; Mansikkamäki, A.; Layfield, R. A., Magnetic hysteresis up to 80 kelvin in a dysprosium metallocene single-molecule magnet. Science 2018, 362 (6421), 1400

(9) Goodwin, C. A. P.; Ortu, F.; Reta, D.; Chilton, N. F.; Mills, D. P., Molecular magnetic hysteresis at 60 kelvin in dysprosocenium. Nature 2017, 548 (7668), 439-442.

(10) Dickie, C. M.; Laughlin, A. L.; Wofford, J. D.; Bhuvanesh, N. S.; Nippe, M., Transition metal redox switches for reversible "on/off" and "slow/fast" single-molecule magnet behaviour in dysprosium and erbium bis-diamidoferrocene complexes. Chem. Sci. 2017, 8 (12), 8039-8049.

(11) Norel, L.; Feng, M.; Bernot, K.; Roisnel, T.; Guizouarn, T.; Costuas, K.; Rigaut, S., Redox Modulation of Magnetic Slow Relaxation in a 4f-Based Single-Molecule Magnet with a 4d Carbon-Rich Ligand. Inorg. Chem. 2014, 53 (5), 2361-2363.

(12) Freedman, D. E.; Jenkins, D. M.; Iavarone, A. T.; Long, J. R., A redox-switchable single-molecule magnet incorporating $\operatorname{Re}(\mathrm{CN})(7)(3-) . J$. Am. Chem. Soc. 2008, 130 (10), 2884-2885.

(13) Liu, J.-L.; Chen, Y.-C.; Zheng, Y.-Z.; Lin, W.-Q.; Ungur, L.; Wernsdorfer, W.; Chibotaru, L. F.; Tong, M.-L., Switching the anisotropy barrier of a single-ion magnet by symmetry change from quasi-D5h to quasi-Oh. Chem. Sci. 2013, 4 (8), 3310-3316.

(14) Tian, H.; Su, J.-B.; Bao, S.-S.; Kurmoo, M.; Huang, X.-D.; Zhang, Y.-Q.; Zheng, L.-M., Reversible ON-OFF switching of single-moleculemagnetism associated with single-crystal-to-single-crystal structural transformation of a decanuclear dysprosium phosphonate. Chem. Sci. 2018, 9, 6424-6433

(15) Jiang, W.; Jiao, C.; Meng, Y.; Zhao, L.; Liu, Q.; Liu, T., Switching single chain magnet behavior via photoinduced bidirectional metal-to-metal charge transfer. Chem. Sci. 2018, 9 (3), 617-622.

(16) Feng, X. W.; Mathoniere, C.; Jeon, I. R.; Rouzieres, M.; Ozarowski, A.; Aubrey, M. L.; Gonzalez, M. I.; Clerac, R.; Long, J. R., Tristability in a Light-Actuated Single-Molecule Magnet. J. Am. Chem. Soc. 2013, 135 (42), 15880-15884

(17) Mathoniere, C.; Lin, H. J.; Siretanu, D.; Clerac, R.; Smith, J. M., Photoinduced Single-Molecule Magnet Properties in a Four-Coordinate Iron(II) Spin Crossover Complex. J. Am. Chem. Soc. 2013, 135 (51), 1908319086.

(18) Cosquer, G.; Breedlove, B. K.; Yamashita, M., Remote control of SMM behaviour via DTE ligands. Dalton Trans. 2015, 44 (7), 2936-2942.

(19) Pinkowicz, D.; Ren, M.; Zheng, L. M.; Sato, S.; Hasegawa, M.; Morimoto, M.; Irie, M.; Breedlove, B. K.; Cosquer, G.; Katoh, K.; Yamashita, M., Control of the Single-Molecule Magnet Behavior of Lanthanide-Diarylethene Photochromic Assemblies by Irradiation with Light. Chem. Eur. J. 2014, 20 (39), 12502-12513.

(20) Yatoo, M.; Cosquer, G.; Morimoto, M.; Irie, M.; Breedlove, B.; Yamashita, M., 1D Chains of Lanthanoid Ions and a Dithienylethene
Ligand Showing Slow Relaxation of the Magnetization. Magnetochemistry 2016, 2 (2), 21

(21) Cosquer, G.; Morimoto, M.; Irie, M.; Fetoh, A.; Breedlove, B. K.; Yamashita, M., Photo-control of the magnetic properties of Dy(iii) and Ho(iii) homometal coordination polymers bridged by a diarylethene ligand. Dalton Trans. 2015, 44 (13), 5996-6002.

(22) Morimoto, M.; Miyasaka, H.; Yamashita, M.; Irie, M., Coordination Assemblies of Mn-4 Single-Molecule Magnets Linked by Photochromic Ligands: Photochemical Control of the Magnetic Properties J. Am. Chem. Soc. 2009, 131 (28), 9823-9835.

(23) Selvanathan, P.; Huang, G.; Guizouarn, T.; Roisnel, T.; FernandezGarcia, G.; Totti, F.; Le Guennic, B.; Calvez, G.; Bernot, K.; Norel, L.; Rigaut, S., Highly Axial Magnetic Anisotropy in a N3O5 Dysprosium(III) Coordination Environment Generated by a Merocyanine Ligand. Chem. Eur. J. 2016, 22 (43), 15222-15226.

(24) Selvanathan, P.; Dorcet, V.; Roisnel, T.; Bernot, K.; Huang, G.; Le Guennic, B.; Norel, L.; Rigaut, S., trans to cis photo-isomerization in merocyanine dysprosium and yttrium complexes. Dalton Trans. 2018, 47 (12), 4139-4148

(25) Fetoh, A.; Cosquer, G.; Morimoto, M.; Irie, M.; El-Gammal, O.; El-Reash, G. A.; Breedlove, B. K.; Yamashita, M., Photo-activation of Single Molecule Magnet Behavior in a Manganese-based Complex. Sci. Rep. 2016, 6, 23785.

(26) Norel, L.; Darago, L. E.; Le Guennic, B.; Chakarawet, K.; Gonzalez, M. I.; Olshansky, J. H.; Rigaut, S.; Long, J. R., A Terminal Fluoride Ligand Generates Axial Magnetic Anisotropy in Dysprosium Complexes. Angew. Chem. Int. Ed. 2018, 57 (7), 1933-1938

(27) Canaj, A. B.; Singh, M. K.; Regincós Marti, E.; Damjanović, M.; Wilson, C.; Céspedes, O.; Wernsdorfer, W.; Rajaraman, G.; Murrie, M. Boosting axiality in stable high-coordinate Dy(iii) single-molecule magnets. Chem. Commun. 2019, 55 (42), 5950-5953.

(28) Gilat, S. L.; Kawai, S. H.; Lehn, J. M., Light-triggered molecular devices - photochemical switching of optical and electrochemical properties in molecular wire type diarylethene species. Chem. Eur. J. 1995, I (5), 275 284.

(29) Irie, M.; Fulcaminato, T.; Matsuda, K.; Kobatake, S., Photochromism of Diarylethene Molecules and Crystals: Memories, Switches, and Actuators. Chem. Rev. 2014, 114 (24), 12174-12277.

(30) Ruiz-Martínez, A.; Casanova, D.; Alvarez, S., Polyhedral Structures with an Odd Number of Vertices: Nine-Coordinate Metal Compounds. Chem. Eur. J. 2008, 14 (4), 1291-1303.

(31) Kobatake, S.; Uchida, K.; Tsuchida, E.; Irie, M., Single-crystalline photochromism of diarylethenes: reactivity-structure relationship. Chem. Commun. 2002, (23), 2804-2805.

(32) Lorenc, M.; Balde, C.; Kaszub, W.; Tissot, A.; Moisan, N.; Servol, M.; Buron-Le Cointe, M.; Cailleau, H.; Chasle, P.; Czarnecki, P.; Boillot, M. L.; Collet, E., Cascading photoinduced, elastic, and thermal switching of spin states triggered by a femtosecond laser pulse in an Fe(III) molecular crystal. Physical Review B 2012, 85 (5), 054302.

(33) Trzop, E.; Buron-Le Cointe, M.; Cailleau, H.; Toupet, L.; Molnar, G.; Bousseksou, A.; Gaspar, A. B.; Real, J. A.; Collet, E., Structural investigation of the photoinduced spin conversion in the dinuclear compound $\{[\mathrm{Fe}(\mathrm{bt})(\mathrm{NCS}) 2] 2(\mathrm{bpym})\}$ : toward controlled multi-stepped molecular switches. J. Appl. Crystallogr. 2007, 40 (1), 158-164.

(34) Morimoto, M.; Irie, M., Photochromism of diarylethene single crystals: crystal structures and photochromic performance. Chem. Commun. 2005, (31), 3895-3905.

(35) Brayshaw, S. K.; Schiffers, S.; Stevenson, A. J.; Teat, S. J.; Warren, M. R.; Bennett, R. D.; Sazanovich, I. V.; Buckley, A. R.; Weinstein, J. A.; Raithby, P. R., Highly Efficient Visible-Light Driven Photochromism: Developments towards a Solid-State Molecular Switch Operating through a Triplet-Sensitised Pathway. Chem. Eur. J. 2011, 17 (16), 4385-4395.

(36) Lunghi, A.; Totti, F.; Sanvito, S.; Sessoli, R., Intra-molecular origin of the spin-phonon coupling in slow-relaxing molecular magnets. Chem. Sci. 2017, 8 (9), 6051-6059.

(37) Lunghi, A.; Totti, F.; Sessoli, R.; Sanvito, S., The role of anharmonic phonons in under-barrier spin relaxation of single molecule magnets. Nat. Commun. 2017, 8, 14620 . 
TOC graphic

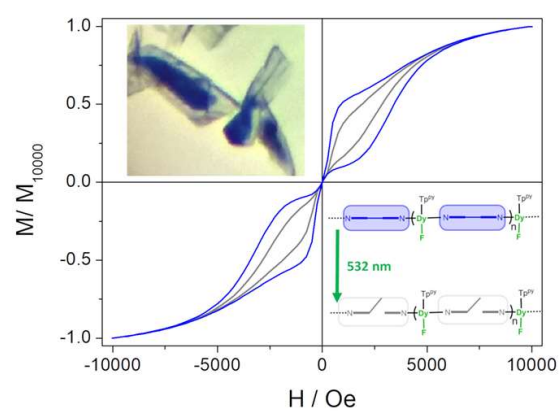

